\title{
Parentalidad y su efecto en la autodeterminación y el bienestar adolescente
}

\author{
Begoña Cordero-López, Mg. ${ }^{\text {a }}$ \\ Universidad de Talca, Chile \\ Joan Calventus-Salvador, $\mathbf{M g} .^{\text {b }}$ \\ Conicet, Argentina
}

mlopezc@utalca.cl

\section{Resumen (analítico)}

El objetivo del estudio fue conocer los efectos de la parentalidad en la autodeterminación y en la calidad de vida de los adolescentes.

Participaron 544 adolescentes escolarizados ( $55 \%$ mujeres), entre los 13 y 18 años. Se analizó el efecto de la parentalidad en la autodeterminación de los adolescentes y en el bienestar subjetivo y psicológico con análisis de mediación. Se obtienen cuatro modelos significativos. Se utilizó el contraste de medidores de idoneidad. Se acepta la hipótesis de relación causal y explicativa de la parentalidad en la autodeterminación y el efecto en el bienestar subjetivo y el bienestar psicológico. Se concluye que la parentalidad incide significativamente en la autonomía y competencia en la adolescencia; sin embargo, la relación de autodeterminación genera un escaso aporte en el bienestar.

\section{Palabras clave}

Parentalidad, adolescencia, autodeterminación, bienestar subjetivo, bienestar psicológico.

\section{Thesauro}

Tesauro Calidad de vida.

\section{Para citar este artículo}

Cordero-López, B., \& Calventus-Salvador, J. (2022). Parentalidad y su efecto en la autodeterminación y el bienestar adolescente. Revista Latinoamericana de Ciencias Sociales, Niñez y Juventud, 20(1), 1-23. https://dx.doi.org/10.11600/rlcsnj.20.1.5118

\section{Historial}

Recibido: 08.10.2020

Aceptado: 01.02.2021

Publicado: 07.12.2021

\section{Información artículo}

Presenta los resultados de la investigación titulada Efecto de los estilos y competencias parentales en el bienestar adolescente y tendencias motivacionales. Se realizó durante el primer semestre de 2020 y fue financiada por la Universidad de Talca. Área: ciencias sociales y humanas. Subárea: parentalidad y calidad de vida adolescente. 


\section{Parenting and its effect on adolescent self-determination and wellbeing}

\section{Abstract (analytical)}

The objective of this research was to determine the effects of parenting on self-determination and quality of life for adolescents. A total of 544 adolescents (55\% female) between 13 and 18 years of age participated in the study. The effect of parenting on adolescent self-determination and subjective and psychological well-being is analyzed using mediation analysis. Four significant models are obtained. The fit-means test was used. The hypothesis of a causal and explanatory relationship of parenting on self-determination and the effect on subjective well-being and psychological wellbeing is accepted. It is concluded that parentality has a significant impact on autonomy and competence in adolescence. However, the self-determination relationship generates a low contribution to well-being.

Keywords

Parentality, adolescence, self-determination, subjective well-being, psychological well-being.

\section{A parentalidade e o seu efeito na autodeterminação e bem-estar dos adolescentes}

\section{Resumo (analítico)}

O objectivo desta investigação era determinar os efeitos da parentalidade na autodeterminação e qualidade de vida dos adolescentes. Um total de 544 adolescentes em idade escolar (55\% mulheres) entre os 13 e 18 anos de idade participaram no estudo. $O$ efeito da parentalidade na autodeterminação dos adolescentes e no seu bem-estar subjectivo e psicológico foi analisado com a análise da mediação. Foram obtidos quatro modelos significativos. Utilizámos o teste de aptidão dos meios. É aceite a hipótese de relação causal e explicativa da parentalidade sobre a autodeterminação e o efeito sobre o bem-estar subjectivo e o bem-estar psicológico. Conclui-se que a parentalidade tem um impacto significativo na autonomia e competência na adolescência; contudo, a relação de autodeterminação gera uma escassa contribuição para o bem-estar.

Palavras-chave

Parentalidade, adolescencia, autodeterminação, bem-estar subjectivo, bem-estar psicológico.

Información autores

[a] Psicóloga y magíster en Diagnóstico y Técnicas de Intervención Psicodinámicas Breves, Universidad del Desarrollo. Profesora de la cátedra Ciclo Vital. (iD) 00000-0001-8326-2601. H5: 0. Correo electrónico:

mlopezc@utalca.cl

[b] Psicólogo y magíster en Sociología, Pontificia Universidad Católica de Chile. (iD) 0000-0002-5781-6794. H5: 0. Correo electrónico: jcalventus@gmail.com 


\section{Introducción}

【 a parentalidad tiene repercusiones en el bienestar, la calidad de vida y la motiEstudios recientes reafirman el impacto que tiene para el desarrollo emocional, cognitivo y la salud mental en la adultez (Huver et al., 2010; Mastrotheodoros et al., 2020; Montes et al., 2019; Oyanedel et al., 2015). La evidencia teórica y empírica muestra de forma sostenida cómo la parentalidad posee una importante injerencia en la personalidad adulta. Sin embargo, las investigaciones en bienestar adolescente son insuficientes, considerando que la mayoría están referidas al área de la salud física (Alfaro et al., 2015; Borrás, 2014; HiguitaGutiérrez \& Cardona-Arias, 2015; Maganto et al., 2019; Steinmayr et al., 2019).

La parentalidad es entendida como la interacción entre los estilos y las prácticas parentales de socialización (Albarracín-León \& Alvarado-León, 2020; Darling \& Steinberg, 1993; Darling \& Toyokawa, 1997) que intervienen en el proceso de desarrollo y tiene una máxima incidencia en el período adolescente con implicancias en el bienestar, la satisfacción vital y la felicidad (Chervonsky \& Hunt, 2019; Molina et al., 2015; Schoenmaker et al., 2015; Wood et al., 2018). En este sentido, el bienestar humano ha sido estudiado por distintos enfoques teóricos y empíricos (Barrera et al., 2019; Díaz et al., 2006; Oishi et al., 2009; Ryff \& Keyes, 1995). Cada enfoque, desde su perspectiva, define y enfatiza la relevancia y las repercusiones que tiene para la vida de las personas, en el aprendizaje, el desarrollo personal y la salud mental (Alfaro et al., 2015; Bilbao et al., 2007; García et al., 2019; Lansford, 2018; Meier \& Oros, 2019). La perspectiva hedónica focaliza sus estudios en el bienestar subjetivo (BS) y lo define como un estado de satisfacción general con la vida o un estado de felicidad. Las personas cognitivamente valoran y evalúan su nivel de satisfacción general, considerando sus estados afectivos, positivos y negativos (Alfaro et al., 2015; Díaz et al., 2011; Moyano \& Ramos, 2007; Oishi et al., 2000; Oishi et al., 2009).

Los estudios sobre el bienestar se sustentan en una fértil base de información y conocimiento respecto de las características personales y contextuales propicias para el 
desarrollo de las personas. Proponen que mientras mayor sea el BS o satisfacción vital, mejor salud, más larga vida e, incluso, vivirían más que las personas con un nivel de salud inferior (Diener \& Chan, 2011; Diener et al., 2017). Por otra parte, la perspectiva de tradición eudaimónica estudia el bienestar psicológico (BP) en relación al desarrollo personal y la búsqueda de trascendencia. En este sentido, el foco central está en el potencial humano y el significado de crecimiento personal. Incorpora áreas o dimensiones que las personas necesitan desarrollar para un buen estado de bienestar (Bilbao, 2008; Chen et al., 2013; Nurius et al., 2015; Quintal \& Flores, 2020; Ryff, 1995), dentro de las cuales se identifican: autoaceptación, relaciones positivas, autonomía, dominio del entorno, crecimiento personal y propósito en la vida.

Disabato et al. (2016) realizan un estudio comparativo entre las variables bienestar subjetivo (hedónico) y bienestar psicológico (eudaimónico), con el objetivo de discriminar si ambas representan un constructo global de bienestar o más bien dimensiones relacionadas. Para esto obtienen una muestra procedente de 109 países de los siete continentes ( $54 \%$ casados y $79 \%$ mujeres). Los resultados sugieren que ambas formas de bienestar reflejan una estructura de bienestar global con una alta correlación $r=.96$. Así mismo, se cuenta con estudios que asocian metas personales (propósito en la vida) y satisfacción vital o BS (Álvarez \& Montoya, 2020; 2020; Castro \& Díaz, 2002; Sánchez, 2019) con satisfacción vital familiar (Cardoza et al., 2019; Luna et al., 2011; Vergaray \& Benavides, 2020), funcionamiento positivo, autorrealización y BP (Delgado \& Tejeda, 2020; GonzálezFuentes \& Andrade, 2016; Gutiérrez-Carmona et al., 2020). En esta misma línea, la teoría de la autodeterminación (TAD, Self-Determination Theory) de Ryan y Deci (2000), se encuentra orientada hacia la motivación personal. Estos autores determinan sine qua non, para el bienestar humano, la satisfacción de las necesidades básicas: autonomía, competencia y relación.

\section{Parentalidad y autodeterminación}

El sentido de gestión de la propia vida es un factor importante en el período de formación adolescente. La propuesta de metas personales y la toma de decisiones se han asociado de forma central a la consecución de un nivel superior de calidad de vida. En este contexto, la familia — a través de la parentalidad- promueve la autodeterminación de sus miembros, especialmente en la adolescencia.

Este es un momento del ciclo vital que requiere se constituya la tarea compleja de la individualización (García et al., 2019; Minchon, 2019; Torres, 2019; Wehmeyer, 2014; Villarejo 
et al., 2020). Existen padres que consideran a sus hijos adolescentes como personas activas, comprometidas y proactivas con sus vidas. En tanto, otros padres se sienten frustrados, al observar la falta de compromiso, la actitud pasiva y alienada de los hijos. Deci y Ryan (1995) señalan, al respecto, que por naturaleza tendemos al crecimiento, la búsqueda y al aprendizaje; no obstante, esta tendencia es frenada por los contextos más cercanos, es decir, la familia y los padres (Ryan \& Deci, 200o; Ryan et al., 2008; Ryan \& Martela, 2016).

Un meta-análisis confirma la asociación entre la necesidad de autonomía y el BS, en apoyo a la propuesta de la TAD, como una necesidad psicológica universal (Yu et al., 2018). La satisfacción de las necesidades básicas de la TAD afectaría el BS y la afectividad en la adolescencia. Un estudio longitudinal realizado por Gnambs y Hanfstingl (2016) revisa el declive de la motivación intrínseca académica en adolescentes de 11 a 16 años. Este estudio muestra cómo las diferencias en la satisfacción de las necesidades psicológicas básicas de la TAD (autonomía, competencia y relación) predicen la trayectoria del cambio motivacional. Khaleque y Rohner (2002) concluyen que, en la adolescencia, son centrales y significativas la consolidación de la identidad personal y la adaptación social.

El objetivo de esta investigación es valorar el impacto de la parentalidad en la calidad de vida adolescente, por ser un momento crucial de hipersensibilidad a los estímulos externos e internos. En Latinoamérica existe escasa literatura científica que revise la parentalidad y el bienestar en relación con la autodeterminación en la adolescencia. Es importante profundizar estas variables y sus efectos por las importantes implicaciones que tienen para el desarrollo integral, la salud mental, la identidad personal (Orcasita et al., 2020; Moreno et al., 2020; Pons et al., 2020; Quiroga et al., 2021; Vergaray \& Benavides, 2020).

En el presente estudio nos proponemos explicar el efecto directo e indirecto de la parentalidad en las necesidades psicológicas básicas de la TAD y los bienestares (BS y $\mathrm{BP})$ en la adolescencia, así como analizar el efecto mediador de las necesidades psicológicas básicas en el bienestar.

Partimos de los siguientes planteamientos: primero, que los adolescentes presentan distintas formas de percibir el BS y el BP y que estos se complementan; segundo, que la parentalidad (estilo y práctica parental) está relacionada con el BS y el BP y, tercero, que la parentalidad tiene un efecto en la autodeterminación adolescente. 


\section{Método}

Para responder a nuestros objetivos se realizó una investigación descriptiva, a través de la aplicación de un diseño transversal correlacional-causal.

\section{Participantes}

Los tres centros educativos seleccionados que accedieron a participar del estudio pertenecen a una zona urbana de la VII región de Chile. Participaron en este estudio un total de 544 jóvenes, con edades comprendidas entre los 13 y los 18 años $(M=15.11, D T=1.052)$, todos ellos escolarizados en colegios científico-humanistas, cursando entre $8^{\circ}$ básico y $3^{\circ}$ medio. De estos, el $22.1 \%$ pertenecían a un colegio particular, el $51.1 \%$ a un colegio subvencionado y el $26.6 \%$ a un colegio público. Las características sociodemográficas de la muestra se presentan en la tabla 1 . Todos los participantes respondieron un cuestionario; 278 de ellos, en su modalidad en línea y 266 en formato impreso.

\section{Tabla 1}

Características sociodemográficas de la muestra ( $N=544)$

\begin{tabular}{lcc}
\hline Variables & Muestra $(\mathbf{n = 5 4 4 )}$ & Porcentaje \\
\hline Unidad Educativa & & \\
Liceo Pablo Neruda & 278 & $51 \%$ \\
Colegio de la Salle & 120 & $22 \%$ \\
Liceo Abate Molina & 146 & $27 \%$ \\
Sexo & & \\
Mujeres & 295 & $54 \%$ \\
Hombres & 249 & $46 \%$ \\
Curso & & \\
$8^{\circ}$ básico & 43 & $7.9 \%$ \\
$1^{\circ}$ medio & 169 & $31.1 \%$ \\
$2^{\circ}$ medio & 193 & $35.5 \%$ \\
$3^{\circ}$ medio & 139 & $25.6 \%$ \\
Con quien vive & & \\
Ambos padres & 344 & $63 \%$ \\
Madre & 162 & $30 \%$ \\
Padre & 16 & $3 \%$ \\
Otros & 22 & $4.1 \%$ \\
NSE & & \\
Homogéneo alto & 120 & $22.1 \%$ \\
Heterogéneo bajo-medio & 278 & $51.1 \%$ \\
Heterogéneo medio-bajo & 146 & $26.8 \%$ \\
\hline
\end{tabular}




\section{Instrumentos}

Escala de satisfacción con la vida (SWLS) de Diener et al. (1985) es una versión traducida al castellano y validada con adolescentes (Atienza et al., 200o). Es una medida subjetiva de bienestar que evalúa el juicio global que la persona da a su propia vida. La escala consta de cinco ítems de tipo Likert (con siete alternativas de respuesta cada uno). La versión española muestra confiabilidad y validez satisfactoria (Cárdenas et al., 2012). La SWLS ha mostrado una alta consistencia interna con alphas que oscilan entre .89 y .79 (Atienza et al., 2000). En este estudio, el coeficiente alfa de Cronbach fue de $(\mathbf{a}=.88)$.

La Escala de bienestar psicológico (Ryff, 1989) fue adaptada por van Dierendonck (2004) y traducida al español por Díaz et al. (2006); consta de 39 ítems y evalúa el bienestar psicológico a través de seis dimensiones: autoaceptación, dominio del entorno, autonomía, propósito en la vida, crecimiento personal y relaciones positivas con otros. En este estudio se incluyen solo las cinco primeras dimensiones (para evitar colinealidad). Los ítems son de tipo Likert, con seis alternativas de respuesta cada uno. La validación de la escala obtuvo una consistencia interna de $\mathbf{a}=.8 \mathrm{o}$. En este estudio, el coeficiente alfa de la escala total fue de $\mathbf{a}=.89$.

La Escala de satisfacción de las necesidades psicológicas básicas en general (BNSG-S; Gagné, 2003), en su adaptación española (González-Cutre et al., 2015), consta de 21 ítems que evalúan la autodeterminación a través de tres dimensiones: competencia (seis ítems, $\mathbf{a}=.72$ ), autonomía (siete ítems, $\mathbf{a}=.81$ ) y relación (ocho ítems, $\mathbf{a}=.82$ ). Los ítems son tipo Likert, con siete alternativas de respuesta cada uno. En este estudio la escala obtuvo un $\mathrm{a}=.88$.

La Escala parental breve (EPB), desarrollada a partir del PSI II (Cumsille et al., 2014), evalúa de manera breve tres dimensiones: responsividad/calidez, demanda/expectativas y práctica parental (monitoreo). El cuestionario consta de doce ítems tipo Likert, con cinco alternativas cada uno. La escala muestra una confiabilidad adecuada, con $\mathbf{a}=.81$ (Cumsille et al., 2014). En este estudio, la escala obtuvo un $\mathbf{a}=.86$.

\section{Procedimiento}

Los cuestionarios fueron administrados a una muestra suficientemente representativa de la VII región de Chile. A partir de esta, se recoge la valoración que tienen los adolescentes de la parentalidad, la calidad de vida percibida y la autodeterminación. Se especifica que se toma la muestra en el tramo etario de 13 a 18 años para recoger la heterogeneidad 
de percepciones y precisamente por estar en pleno desarrollo el pensamiento abstracto y la construcción de su identidad.

Los cuestionarios se aplicaron en tres centros educativos, durante el horario de clases. En el caso del formato impreso, una persona del equipo con formación especializada en psicología administró el protocolo. En el caso del formato digital se utilizó la plataforma de formularios de Google. El tiempo promedio utilizado para responder el instrumento osciló entre 15 y 20 minutos.

El estudio contó con la aprobación del Comité de Ética Científica de la Universidad de Talca; en este sentido, cumplió los criterios éticos de investigación con seres humanos (consentimiento informado y derecho de información, protección de datos personales y garantías de confidencialidad, no discriminación, gratuidad y posibilidad de abandonar el estudio en cualquiera de sus fases).

\section{Análisis de datos}

En primer lugar, se analizó descriptivamente la muestra a través de los indicadores de Medias, DE, alfa de Cronbach y las correlaciones entre variables. Además, se realizaron análisis de comparación de medias (t-student) para comprobar las diferencias en función del tipo de administración del cuestionario. Los resultados indicaron que no existen diferencias estadísticamente significativas entre la aplicación del cuestionario en papel y la aplicación en línea. En segundo lugar, con el fin de establecer asociación entre las variables del estudio, se realizó un análisis de correlaciones de Pearson (r). En tercer lugar, se efectuaron análisis de mediación simple y múltiple para comparar los efectos directos, indirectos y totales de la parentalidad (en adelante P) con los bienestares (BS y BP) y las dimensiones de la TAD. Se analizan cuatro modelos de mediación múltiple. Por último, se valoró la idoneidad de los modelos a través del contraste por medio de la comparación entre mediadores. Todos estos análisis se realizaron con el software SPSS Windows (versión 23) y Process 3.1.

\section{Resultados}

La tabla 2 muestra un análisis descriptivo de las variables del estudio, así como las correlaciones entre ellas. Se destacan altas correlaciones entre parentalidad y monitoreo ( $\mathrm{p}=. \mathrm{oo1}$ ), entre P y responsividad/calidez ( $\mathrm{p}=. \mathrm{oo1})$, así como entre Comp-TAD y BP ( $\mathrm{p}=$ .o01). Todas las alphas se encuentran sobre $\mathbf{a}=.73$. 
Tabla 2

Medias, DS, Cronbach Alpha y correlaciones entre las variables del estudio

\begin{tabular}{|c|c|c|c|c|c|c|c|c|c|c|c|c|c|c|}
\hline & $M$ & DS & a & 1 & 2 & 3 & 4 & 5 & 6 & 7 & 8 & 9 & 10 & 11 \\
\hline 1. Parentalidad (P) & 49.66 & 7.13 & .86 & 1 & & & & & & & & & & \\
\hline $\begin{array}{l}\text { 2.Responsividad/ } \\
\text { calidez }\end{array}$ & 17.05 & 3.20 & .88 & $.868^{\star \star}$ & 1 & & & & & & & & & \\
\hline $\begin{array}{l}\text { 3.Demanda/ } \\
\text { expectativa }\end{array}$ & 15.98 & 1.62 & .85 & $.759^{\star \star}$ & $.574^{\star \star}$ & 1 & & & & & & & & \\
\hline 4. Monitoreo & 16.64 & 3.54 & .84 & $.883^{\star \star}$ & $.582^{\star}$ & $.552^{\star}$ & 1 & & & & & & & \\
\hline 5. BS & 22.49 & 6.69 & .88 & $.390^{\star \star}$ & $.391^{* *}$ & $.212^{\star *}$ & $.336^{\star \star}$ & 1 & & & & & & \\
\hline $\begin{array}{l}\text { 6. Autonomía TAD } \\
\text { Aut-TAD }\end{array}$ & 33.86 & 7.52 & .74 & $.345^{\star \star}$ & $.450^{\star \star}$ & $.224^{\star \star}$ & $.186^{\star \star}$ & $.431^{\star \star}$ & 1 & & & & & \\
\hline $\begin{array}{l}\text { 7. Competencia TAD } \\
\text { Comp-TAD }\end{array}$ & 28.66 & 6.87 & .73 & $.376^{\star \star}$ & $.438^{\star \star}$ & $.227^{\star \star}$ & $.257^{\star \star}$ & $.408^{\star \star}$ & $.631^{\star \star}$ & 1 & & & & \\
\hline $\begin{array}{l}\text { 8. Relación TAD } \\
\text { Rel-TAD }\end{array}$ & 43.00 & 8.20 & .80 & $.342^{\star \star}$ & $.399^{\star \star}$ & $.228^{\star \star}$ & $.223^{\star \star}$ & $.374^{\star \star}$ & $.659^{\star \star *}$ & $.649 * \star$ & 1 & & & \\
\hline 9. BP & 166.25 & 25.65 & .89 & $.463^{\star \star}$ & $.528^{\star \star}$ & $.303^{* *}$ & $.317^{\star \star}$ & $.585^{\star \star}$ & $.720^{\star \star}$ & $.773^{\star \star}$ & $.696^{\star}$ & 1 & & \\
\hline $\begin{array}{l}\text { 10.Crecimiento BP } \\
\text { Crep-BP }\end{array}$ & 32.48 & 5.84 & .73 & $.416^{\star \star}$ & $.460^{\star \star}$ & $.297^{\star \star}$ & $.285^{\star \star}$ & $.512^{\star \star}$ & $.624^{\star \star}$ & $.712^{\star \star}$ & $.596^{\star \star}$ & $.866^{\star \star}$ & 1 & \\
\hline $\begin{array}{l}\text { 11. Propósito BP } \\
\text { Prop-BP }\end{array}$ & 26.38 & 6.21 & .80 & $.400^{\star \star}$ & $.434^{\star \star}$ & $.272^{\star \star}$ & $.289^{\star \star}$ & $.562^{\star \star}$ & $.533^{\star \star}$ & $.674^{\star \star}$ & $.522^{\star \star}$ & $.843^{\star \star}$ & $.684^{* *}$ & 1 \\
\hline
\end{tabular}

Nota. ${ }^{* *} \mathrm{p}<.01 ;{ }^{*} \mathrm{p}<.05$

Para los análisis de mediación se utilizó el estadístico del modelo 6 de Process (Hayes, 2018). Se obtuvieron cuatro modelos de mediación. Estos corroboraron el efecto directo de la P en el BS (figura 1), además de los efectos indirectos de las regresiones (tabla 3) y los contrastes, entendiendo que la P es mediada por la Aut-TAD con un efecto en la comp-TAD, que impacta positivamente el BS adolescente.

En el primer modelo (P y BS), además del efecto directo $(\mathrm{P} \rightarrow \mathrm{BS})$, observamos tres efectos indirectos (figura 1): 1) P $\rightarrow$ Aut-TAD $\rightarrow$ BS; 2) P $\rightarrow$ Aut-TAD $\rightarrow$ Comp-TAD $\rightarrow$ BS; y 3$) \mathrm{P} \rightarrow$ Comp-TAD $\rightarrow$ BS. Como puede comprobarse en la tabla 3, todos estos efectos son significativos. En dicha tabla también puede observarse que, según el análisis de contrastes, el efecto indirecto $\mathrm{P} \rightarrow \mathrm{Aut}-\mathrm{TAD} \rightarrow \mathrm{BS}$ es más significativo que el efecto indirecto $\mathrm{P} \rightarrow$ Aut-TAD $\rightarrow$ Comp-TAD $\rightarrow$ BS (.o518); y que el efecto indirecto $\mathrm{P} \rightarrow$ Aut-TAD $\rightarrow$ BS es significativamente mayor que $\mathrm{P} \rightarrow$ Comp-TAD $\rightarrow$ BS (.0492). La mediación de Aut-TAD es la que presenta el efecto más significativo. 
Figura 1

Modelo de mediación 1 P y BS

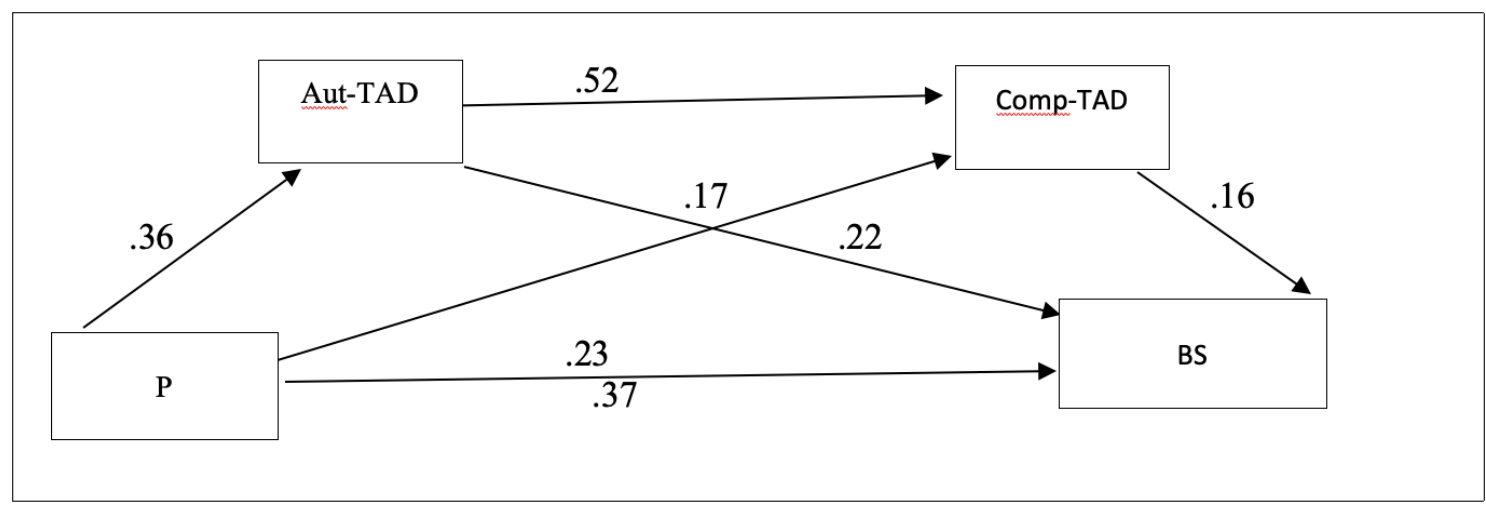

Tabla 3

Efectos y contrastes del modelo de mediación 1 P y BS

\begin{tabular}{lll}
\hline Regresiones y contrastes & Efecto & IC (95\%) \\
$\mathrm{P} \rightarrow \mathrm{BS}$ & .1362 & {$[.0934-.1883]^{*}$} \\
$\mathrm{P} \rightarrow \mathrm{Aut}-\mathrm{TAD} \rightarrow \mathrm{BS}$ & .0791 & {$[.0445-.1207]^{\star}$} \\
$\mathrm{P} \rightarrow$ Aut-TAD $\rightarrow$ Comp-TAD $\rightarrow$ BS & .0273 & {$[.0086-.0513]^{\star}$} \\
$\mathrm{P} \rightarrow$ Comp-TAD $\rightarrow$ BS & .0299 & {$[.0104-.0540]^{*}$} \\
$\mathrm{C} 1 \mathrm{P} \rightarrow$ Aut-TAD $\rightarrow$ BS $>\mathrm{P} \rightarrow$ Aut-TAD $\rightarrow$ Comp-DT $\rightarrow$ BS & .0518 & {$[.0052-.1032]$} \\
$\mathrm{C} 2 \mathrm{P} \rightarrow$ Aut-TAD $\rightarrow$ BS $>\mathrm{P} \rightarrow$ Comp-TAD $\rightarrow$ BS & .0492 & {$[.0039-.0978]$} \\
$\mathrm{C} 3: \mathrm{P} \rightarrow$ Aut-TAD $\rightarrow$ Comp-TAD $\rightarrow \mathrm{BS}=\mathrm{P} \rightarrow$ Comp-TAD $\rightarrow$ BS & -.0026 & {$[-.0221-.0142] \mathrm{ns}$} \\
\hline
\end{tabular}

Nota. * Efecto significativo; ns: no significación estadística

En el segundo modelo ( $\mathrm{P}$ y $\mathrm{BP})$, además del efecto directo $(\mathrm{P} \rightarrow \mathrm{BP})$, observamos tres efectos indirectos (figura 2): 1) $\mathrm{P} \rightarrow$ Aut-TAD $\rightarrow \mathrm{BP}$; 2) $\mathrm{P} \rightarrow$ Aut-TAD $\rightarrow$ Comp-TAD $\rightarrow$ BP; y 3) $\mathrm{P} \rightarrow$ Comp-TAD $\rightarrow$ BP. Como puede comprobarse en la tabla 4 , todos estos efectos son significativos. En dicha tabla, el análisis de contrastes indica que ninguno de estos efectos es significativamente mayor que los demás. 
Figura 2

Modelo de mediación 2 P y BP

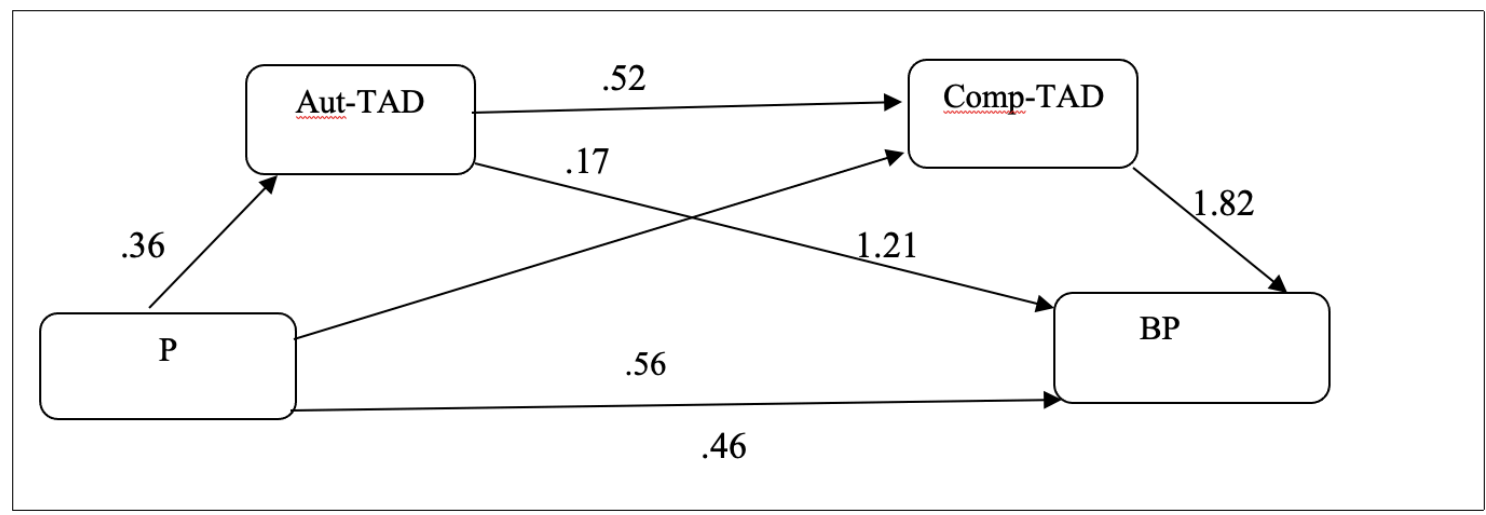

Tabla 4

Efectos y contrastes del modelo 2 de mediación P y BP

\begin{tabular}{|c|c|c|}
\hline Regresiones y contrastes & Efecto & IC (95 \%) \\
\hline $\mathrm{P} \rightarrow \mathrm{BP}$ & 1.1060 & {$[.8472-1.4185]$ * } \\
\hline $\mathrm{P} \rightarrow$ Aut-TAD $\rightarrow$ BP & .4440 & {$[.3013-.6172]^{*}$} \\
\hline $\mathrm{P} \rightarrow$ Aut-TAD $\rightarrow$ Comp-TAD $\rightarrow$ BP & .3158 & {$[.1793-.4728]^{*}$} \\
\hline $\mathrm{P} \rightarrow \mathrm{Comp}-\mathrm{TAD} \rightarrow \mathrm{BP}$ & .3452 & {$[.2316-.4838]^{\star}$} \\
\hline $\mathrm{C} 1: \mathrm{P} \rightarrow$ Aut-TAD $\rightarrow \mathrm{BP}=\mathrm{P} \rightarrow$ Aut-TAD $\rightarrow$ Comp-TAD $\rightarrow$ BP & .1282 & {$[-.0952-.3563] \mathrm{ns}$} \\
\hline $\mathrm{C} 2: \mathrm{P} \rightarrow$ Aut-TAD $\rightarrow \mathrm{BP}=\mathrm{P} \rightarrow$ Comp-TAD $\rightarrow \mathrm{BP}$ & .0978 & {$[-.0289-.2313] \mathrm{ns}$} \\
\hline C3: $\mathrm{P} \rightarrow$ Aut-TAD $\rightarrow$ Comp-TDA $\rightarrow$ BP $=\mathrm{P} \rightarrow$ Comp-TAD $\rightarrow$ BP & -.0304 & {$[-.2244-.1584] \mathrm{ns}$} \\
\hline
\end{tabular}

Nota. * Efecto significativo; ns: no significación estadística

En el tercer modelo (P y Crep-BP), además del efecto directo ( $\rightarrow$ Crep-BP), observamos tres efectos indirectos (figura 3): 1) $\mathrm{P} \rightarrow$ Aut-TAD $\rightarrow$ Crep-BP; 2) $\mathrm{P} \rightarrow$ Aut-TAD $\rightarrow$ Comp-TAD $\rightarrow$ Crep-BP; y 3) P $\rightarrow$ Comp-TAD $\rightarrow$ Crep-BP. Como puede observarse en la tabla 4 , todos estos efectos son significativos. El análisis de contrastes nos muestra que ninguno de estos efectos es significativamente mayor que los demás (tabla 5 ). 
Figura 3

Modelo de mediación 3 P y Crep-BP

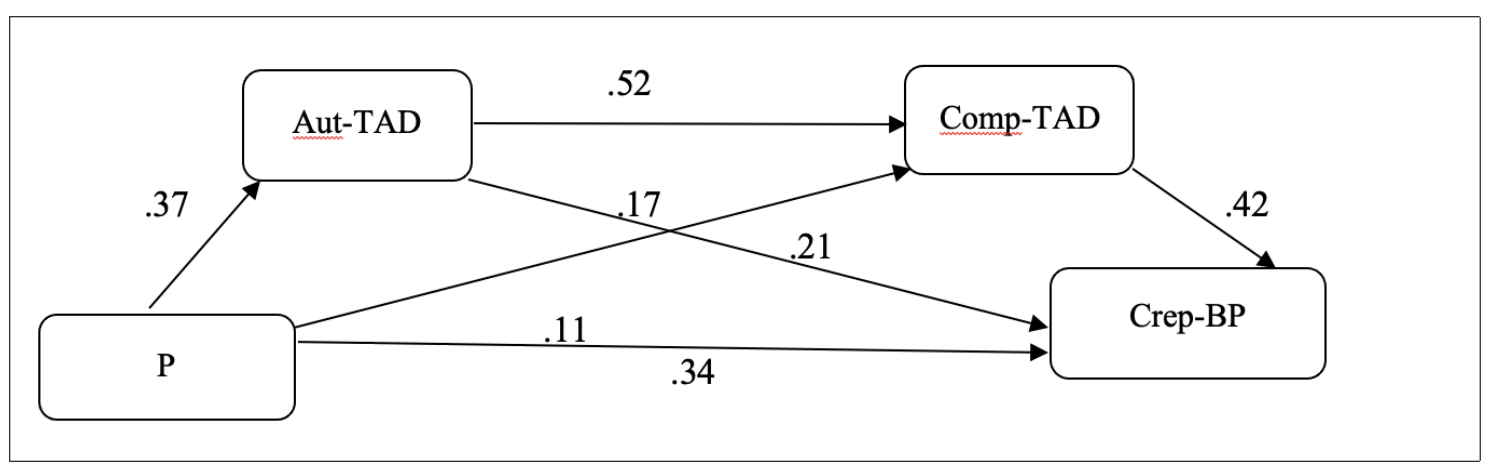

Tabla 5

Efectos y contrastes del modelo de mediación 3 P y Crep-BP

\begin{tabular}{lll}
\hline Regresiones y contrastes & Efecto & IC (95 \%) \\
$P \rightarrow$ Crep-BP & .2269 & {$[.1714-.2934]^{*}$} \\
$\mathrm{P} \rightarrow$ Aut-TAD $\rightarrow$ Crep-BP & .0751 & {$[.0453-.1114]^{*}$} \\
$\mathrm{P} \rightarrow$ Aut-TAD $\rightarrow$ Comp-TAD $\rightarrow$ Crep-BP & .0724 & {$[.405-.1064]^{*}$} \\
$\mathrm{P} \rightarrow$ Comp-TAD $\rightarrow$ Crep-BP & .0794 & {$[.0522-.1132] *$} \\
$\mathrm{C} 1: \mathrm{P} \rightarrow$ Aut-TAD $\rightarrow$ Crep-BP $=\mathrm{P} \rightarrow$ Aut-TAD $\rightarrow$ Comp-TAD $\rightarrow$ Crep-BP & .0027 & {$[-.0451-.0539] \mathrm{ns}$} \\
$\mathrm{C} 2: \mathrm{P} \rightarrow$ Aut-TAD $\rightarrow$ Crep-BP $=\mathrm{P} \rightarrow$ Comp-DT $\rightarrow$ Crep-BP & -.0043 & {$[-.0417-.0299] \mathrm{ns}$} \\
$\mathrm{C} 3: \mathrm{P} \rightarrow$ Aut-TDA $\rightarrow$ Comp-TAD $\rightarrow$ Crep-BP $=\mathrm{P} \rightarrow$ Comp-TAD $\rightarrow$ Crep-BP & -.0070 & {$[-.0548-.0346] \mathrm{ns}$} \\
\hline
\end{tabular}

Nota. * Efecto significativo; ns: no significación estadística

En el cuarto modelo (P y Prop-BP), además del efecto directo ( $\mathrm{P} \rightarrow$ Prop-BP), observamos (figura 4) tres efectos indirectos: 1) $\mathrm{P} \rightarrow$ Aut-TAD $\rightarrow$ Prop-BP; 2) P $\rightarrow$ Aut-TAD $\rightarrow$ Comp-TAD $\rightarrow$ Prop-BP; y 3 ) P $\rightarrow$ Comp-TAD $\rightarrow$ Prop-BP. Como puede comprobarse en la tabla 6 , todos estos efectos son significativos. El análisis de contrastes nos muestra que ninguno de estos efectos es significativamente mayor que los demás. 
Figura 4

Modelo de mediación 4 P y Prop-BP

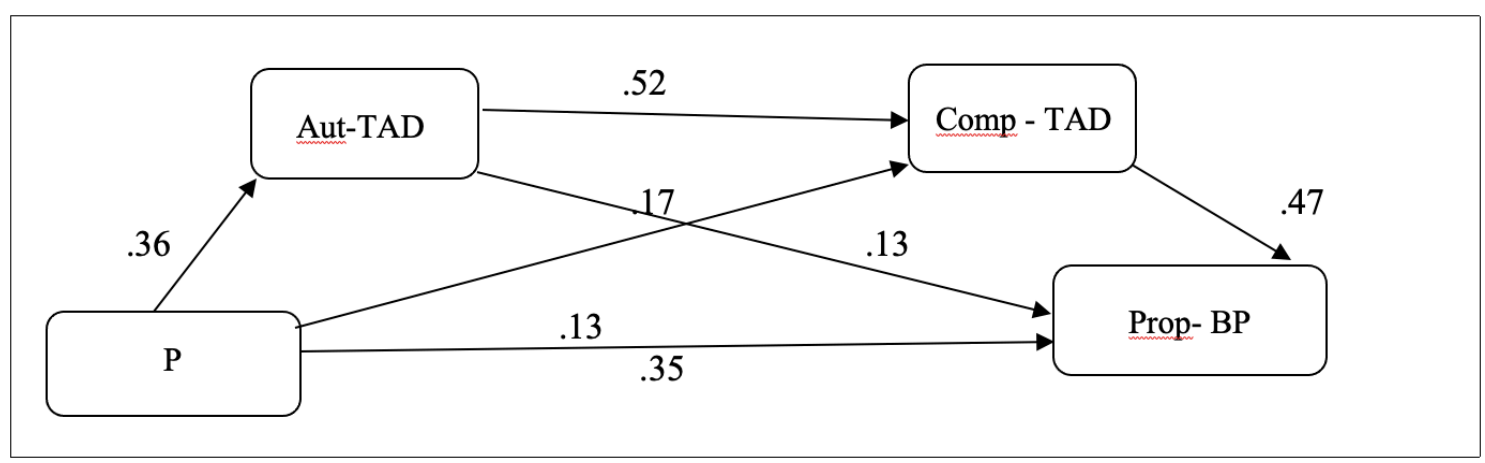

Tabla 6

Efectos y contrastes del modelo de mediación 4 P y Prop-BP

\begin{tabular}{lll}
\hline Regresiones $y$ contrastes & Efecto & IC (95 \%) \\
$P \rightarrow$ Prop-BP & .2165 & {$[.1623-.2811]^{*}$} \\
$P \rightarrow$ Aut-TAD $\rightarrow$ Prop-BP & .0456 & {$[.0178-.0766]^{*}$} \\
$P \rightarrow$ Aut-TAD $\rightarrow$ Comp-TAD $\rightarrow$ Prop-BP & .0816 & {$[.0456-.1219] *$} \\
$P \rightarrow$ Comp-TAD $\rightarrow$ Prop-BP & .0894 & {$[.0586-.1269] *$} \\
$C 1: P \rightarrow$ Aut-TAD $\rightarrow$ Prop-BP $=P \rightarrow$ Aut-TAD $\rightarrow$ Comp-TAD $\rightarrow$ Prop-BP & -.0360 & {$[-.0887-.0121] \mathrm{ns}$} \\
$C 2: P \rightarrow$ Aut-TAD $\rightarrow$ Prop-BP $=P \rightarrow$ Comp-TDA $\rightarrow$ Prop-BP & -.0439 & {$[-.0926-.0054] \mathrm{ns}$} \\
$C 3: P \rightarrow$ Aut-TAD $\rightarrow$ Comp-TAD $\rightarrow$ Prop-BP $=P \rightarrow$ Comp-TDA $\rightarrow$ Prop-BP & -.0079 & {$[-.0598-.0398] \mathrm{ns}$} \\
\hline
\end{tabular}

Nota. * Efecto significativo; ns: no significación estadística

\section{Discusión}

Los objetivos de esta investigación eran valorar el efecto directo e indirecto de la parentalidad en la calidad de vida adolescente, entendiendo esta como resultado del bienestar subjetivo (SB) y el bienestar psicológico (BP). Además, observar el efecto de la parentalidad en las necesidades psicológicas básicas (autonomía, competencia y relación) de la teoría de la autodeterminación y cómo estas podrían impactar el bienestar adolescente.

Primero confirmamos el efecto directo de la parentalidad en la autodeterminación adolescente, específicamente en la autonomía y la competencia. En este sentido, los conflictos relacionados con la necesidad de autonomía entre padres e hijos son altamente 
esperables, normativos (Alonso-Stuyck \& Aliaga, 2017) y son considerados necesarios para el desarrollo, siempre que se mantengan en un contexto de comunicación y confianza. Por otra parte, los resultados obtenidos son congruentes con otras investigaciones que relacionan la autonomía como una necesidad básica universal (Deci \& Ryan, 1995; Ryan \& Martela, 2016), además de ser un indicador de bienestar personal.

A su vez, la autonomía generaría un efecto de satisfacción personal que podría ser incrementado con el sentido de competencia en el adolescente al experimentar sus propias vivencias y poder tomar decisiones. En este sentido, la necesidad de autonomía viene dada por la urgencia en la construcción de un yo más integrado, que necesita reafirmación interna (íntima) y social. Este efecto directo de la parentalidad en la autodeterminación adolescente explica en parte el proceso de integración de la identidad, la necesidad de autonomía y el sentido de competencia, necesarias para gestionar su historia personal, independiente de los padres.

A modo de síntesis, concluimos que las necesidades psicológicas básicas de la TAD cumplen un efecto mediador significativo entre la parentalidad, el bienestar psicológico y subjetivo en los adolescentes. Estos hallazgos son un aporte a la comprensión del complejo proceso de transformación adolescente, que le lleva a experimentar profundos conflictos íntimos y sociales, así como una forma de representar el mundo (Viejo et al., 2013; Bernal-Ruiz et al., 2018; Bonet et al., 2020). La adolescencia es entendida como parte de una crisis normativa, que explica transformaciones hacia la vida adulta. Además, una forma de responder al sistema establecido y coadyuvante al proceso de cambio personal y social, y el resultado de múltiples contextos en los que su identidad se desarrolla (Feixa, 2020). Todas estas aproximaciones teóricas de la adolescencia conducen a la relación con otros significados que se encuentran escasamente estudiados en Latinoamérica. Quiroga et al. (2021) sostienen que la información aportada hasta ahora sobre los aspectos relevantes del desarrollo en contexto de vulneración en la adolescencia es insuficiente. Son variados los estudios que afirman la relevancia de profundizar variables parentales, sus relaciones y efectos (Espinoza-García, 2020; Fernández et al., 2021; Orcasita et al., 2020).

En conclusión, la parentalidad incide especialmente en la autonomía del adolescente, por sobre la competencia de la TAD, y la variable relación no aportaría de forma importante en los efectos de los bienestares BS y BP (no se incluyen en los modelos).

Se aceptan las hipótesis del efecto directo de la parentalidad en el bienestar subjetivo y psicológico, específicamente en las dimensiones de crecimiento y propósito en la vida. Estas dimensiones del BP están muy relacionadas con el conocimiento personal necesario 
para desarrollar el propio potencial y tener una actitud positiva frente a los desafíos personales, además de confianza en planear metas personales y la posibilidad de alcanzarlas (González-Fuentes \& Andrade, 2016; Morales-Castillo et al., 2019).

Por otra parte, se comprueba que los cuatro modelos presentan efectos directos e indirectos significativos: en el primer modelo, la parentalidad tiene un efecto directo sobre el bienestar subjetivo. Esto nos permite confirmar la relevancia de los padres en el estilo de vida saludable de los hijos y que los adolescentes van tendiendo una evaluación crítica de la satisfacción vital (Garuz et al., 2015; Oyanedel et al., 2015; Tirado \& Quezada, 2019). Además, la parentalidad posee un efecto indirecto al ser mediada por la autonomía y la competencia, siendo significativamente mayor la autonomía por sobre la competencia.

En el segundo modelo, la parentalidad tiene un efecto directo en el bienestar psicológico definido por Keyes et al. (2002) como: «la percepción de compromiso con los desafíos existenciales de la vida» (p. 1007), siendo todas significativas: autonomía, crecimiento personal, dominio del medio, relaciones positivas con otros, propósito en la vida y autoaceptación (Díaz et al., 2006; Ryff \& Keyes, 1995). El efecto indirecto de la parentalidad mediada por la autonomía y la competencia serían ambas significativas en el BP.

En el tercer modelo, la parentalidad tiene un efecto directo significativo con el crecimiento personal; además, existe un efecto indirecto significativo entre la parentalidad mediada por la autonomía y la competencia. Mientras que en el cuarto modelo, la parentalidad tiene un efecto directo significativo con el propósito en la vida; así como un efecto indirecto mediado por la autonomía y la competencia, también significativo. cabe destacar que el efecto indirecto de la parentalidad con mayor impacto en los bienestares BS y BP está generado por la mediación de la Aut-TAD.

Los hallazgos son congruentes con los aportes de otras investigaciones de parentalidad y bienestar (Bonet et al., 2020; García et al., 2019; Krabbenborg et al., 2017; Lansford, 2018). Bastidas et al. (2009) identificaron que las relaciones afectivas familiares tenían una importante influencia en la competencia social y la calidad de vida en adolescentes. Por otra parte, Bonet et al. (2020) plantearon que la regulación emocional es aprendida en las relaciones íntimas entre padres e hijos. Además, que los adolescentes carentes de lazo parental tienden a sentirse ineficaces y poco competentes en la vida. En esta línea, Krabbenborg et al. (2017) expusieron que las personas que han vivido el rechazo de sus padres o entornos de crianza inadecuados, presentan desventajas en el desarrollo de la autodeterminación y esto afectaría su bienestar. 
Asimismo, Lansford (2018) concluyó que el desarrollo positivo de los jóvenes se relaciona con las relaciones entre padres e hijos, factor crucial para la adaptación y para el aumento del bienestar subjetivo a lo largo del tiempo. Igualmente, Páez et al. (2006) realizaron un estudio con muestras en tres países (España, México y Chile) para observar el efecto del apego en el bienestar subjetivo y en la inteligencia emocional en la adultez. En sus hallazgos plantean que la persona feliz o aquella que evalúa satisfactoriamente su vida recordaba relaciones con otros significativos como cercana y cálida, y con menos sobreprotección de sus padres en la adolescencia. En otro estudio, Rodríguez-Menéndez et al. (2018) revisaron el efecto de la autodeterminación en la personalidad adolescente y el impacto de la parentalidad en la personalidad. Un factor relevante de análisis es la autonomía, variable que permite el buen desarrollo del ser humano. Es una necesidad básica que requiere ser desplegada en la adolescencia y necesaria en todas las edades del ciclo vital adulto.

Consideramos que este estudio presenta limitaciones en relación con la población o universo de referencia por su limitado alcance local y la necesidad de ampliarlo a otras regiones del país y establecer distinciones entre poblaciones de adolescentes rurales y urbanas, así como incorporar la población de adolescentes inmigrantes, que permitan análisis comparativos transculturales. Por otra parte, incorporar a los análisis los tipos de familias emergentes, las cuales pudiesen enriquecer los resultados obtenidos. Además, incorporar la experiencia de los padres y la percepción de su parentalidad aportaría en información valiosa a explorar y contrastar.

Finalmente, es relevante crear áreas de investigación que favorezcan, profundicen y amplíen el conocimiento de los efectos que tiene la parentalidad en la calidad de vida de los niños y adolescentes, específicamente aquellos que dicen relación con la autonomía y el sentido de competencia, el crecimiento personal y el propósito en la vida. La indagación de estas variables serán un aporte valioso al análisis de la parentalidad en los adolescentes, además de ser una fuente riquísima de nuevo conocimiento para ser incorporado en programas de intervención focalizados en adolescencia, padres, familia y bienestar.

\section{Agradecimientos}

Se agradece al Grupo de Investigación CCE de la Universidad del País Vasco. 


\section{Referencias}

Albarracín-León, M. C. \& Alvarado-León, M. C. (2020). Estilos parentales de crianza y la presencia de conductas internalizantes y externalizantes en adolescentes con un rendimiento académico igual o superior a 9 (domina los aprendizajes) [Tesis de pregrado, Universidad del Azuay]. Repositorio institucional Universidad del Azuay. https://bit.ly/3CtItgS

Alfaro, J., Casas, F. \& López, V. (2015). Bienestar en la infancia y adolescencia [Editorial]. Psicoperspectivas, Individuo y Sociedad, 14(1), 1-5. https://doi.org/g54s

Alonso-Stuyck, P., \& Aliaga, F. M. (2017). Demanda de autonomía en la relación entre los adolescentes y sus padres. Estudios sobre educación, 33. https://doi.org/g53w

Álvarez, M. M., \& Montoya, A. M. (2020). Percepciones de los estudiantes frente a la satisfacción personal desde el enfoque del desarrollo humano. Cultura, Educación y Sociedad, 11(2), 43-56. https://doi.org/10.17981/cultedusoc.11.2.2020.03

Atienza, F., Pons, D., Balaguer, I., \& García-Merita, M. (2000). Propiedades psicométricas de la Escala de Satisfacción con la Vida en adolescentes. Psicothema, 12(2), 314-319.

Barrera, L. F., Sotelo, M. A., Barrera, R. A., \& Aceves, J. (2019). Bienestar psicológico y rendimiento académico en estudiantes universitarios. Enseñanza e Investigación en Psicología, 1(2), 244-251.

Bastidas, H., Carrillo, S., Cabrera, V., \& Ripoll, K. (2009). Relaciones familiares, calidad de vida y competencia social en adolescentes y jóvenes colombianos. Repositorio Universidad del Rosario. https://repository.urosario.edu.co/handle/10336/26874

Bernal-Ruiz, F., Rodríguez-Vera, M., González-Campos, J., \& Torres-Álvarez, A. (2018). Competencias parentales que favorecen el desarrollo de funciones ejecutivas en escolares. Revista Latinoamericana de Ciencias Sociales, Niñez y Juventud, 16(1), 163-176. https://doi.org/10.1160o/1692715x.16109

Bilbao, Á. (2008). Creencias sociales y bienestar: valores, creencias básicas, impacto de los hechos vitales y crecimiento psicológico [Tesis doctoral]. Universidad del País Vasco.

Bilbao, M. Á., Techio, E., \& Páez, D. (2007). Felicidad, cultura y valores personales: estado de la cuestión y síntesis meta-analítica. Revista de Psicología (Lima), 25(2), 135-276. https://doi.org/10.1880o/psico.200702.005

Bonet, C., Palma, C., \& Gimeno-Santos, M. (2020). Riesgo de suicidio, inteligencia emocional y necesidades psicológicas básicas en adolescentes tutelados en centros residenciales. Revista de Psicología Clínica con Niños y Adolescentes, 7(1), 30-37. https:// doi.org/10.21134/rpcna.2020.07.1.4 
Borrás, T. (2014). Adolescencia: Definición, vulnerabilidad y oportunidad. Correo Científico Médico, 18(1), 05-07.

Cárdenas, M., Barrientos, J., Bilbao, Á., Paez, D., Gómez, F., \& Asún, D. (2012). Estructura factorial de la escala de satisfacción con la vida en una muestra de estudiantes universitarios chilenos. Revista mexicana de Psicología, 29(2), 157-164.

Cardoza, M., Hidalgo, L. I., Peña, J., Pulido, L. A., \& Torres, C. G. (2019). Satisfacción con la vida y satisfacción laboral. UCV-Hacer, Revista de Investigación y Cultura, 8(1), 74-89.

Castro, A., \& Díaz, J. F. (2002). Objetivos de vida y satisfacción vital en adolescentes españoles y argentinos. Psicothema, 14(1) 112-117.

Chen, F. F., Jing, Y., Hayes, A., \& Lee, J. M. (2013). Two concepts or two approaches? A bifactor analysis of psychological and subjective well-being. Journal of Happiness Studies, 14(3), 1033-1068. https://doi.org/10.1007/s10902-012-9367-x

Chervonsky, E., \& Hunt, C. (2019). Emotion regulation, mental health, and social wellbeing in a young adolescent sample: A concurrent and longitudinal investigation. Emotion, 19(2), 270-282. https://doi.org/10.1037/emoooo0432

Cumsille, P., Loreto, M., Rodríguez, V., \& Darling, N. (2014). Análisis psicométrico de la Escala Parental Breve (EPB): Invarianza demográfica y longitudinal en adolescentes chilenos. Psykhe (Santiago), 23(2), 1-14.

Darling, N. \& Steinberg, L. (1993). Parenting style as context: An integrative model. Psychological Bulletin, 113(3), 487-496. https://doi.org/10.1037/0033-2909.113.3.487

Darling, N., \& Toyokawa, T. (1997). Construction and validation of the parenting style inventory II (PSI-II) [Manuscrito no publicado]. The Pennsylvania State University.

Deci, E. L., \& Ryan, R. M. (1995). Human autonomy. En M. H. Kernis (Ed.), Efficacy, agency, and self-esteem (pp. 31-49). Springer. https://doi.org/10.1007/978-1-4899-1280-0_3

Delgado, A. M., \& Tejeda, M. C. (2020). Bienestar psicológico en estudiantes universitarios de latinoamérica [Tesis de pregrado, Universidad Cooperativa de Colombia]. Repositorio Institucional UCC. http://hdl.handle.net/20.500.12494/16343

Díaz, D., Blanco, A., \& Durán, M. (2011). La estructura del bienestar: el encuentro empírico de tres tradiciones. Revista de Psicología Social, 26(3), 357-372. https://doi.org/dfqnnd

Díaz, D., Rodríguez-Carvajal, R., Blanco, A., Moreno-Jiménez, B., Gallardo, I., Valle, C., \& van Dierendonck, D. (2006). Adaptación española de las escalas de bienestar psicológico de Ryff. Psicothema, 18(3), 572-577.

Diener, E. D., Emmons, R. A., Larsen, R. J., \& Griffin, S. (1985). The satisfaction with life scale. Journal of Personality Assessment, 49(1), 71-75. https://doi.org/fqqbmr 
Diener, E., \& Chan, M. Y. (2011). Happy people live longer: Subjective well-being contributes to health and longevity. Applied Psychology: Health and Well-Being, 3(1), 1-43. https://doi.org/10.1111/j.1758-0854.2010.01045.x

Diener, E., Pressman, S. D., Hunter, J., \& Delgadillo-Chase, D. (2017). If, why, and when subjective well-being influences health, and future needed research. Applied Psychology: Health and Well-Being, 9(2), 133-167. https://doi.org/10.1111/aphw.12090

Disabato, D. J., Goodman, F., Kashdan, T. B., Short, J. L., \& Jarden, A. (2016). Different types of well-being? A cross-cultural examination of hedonic and eudaimonic wellbeing. Psychological assessment, 28(5), 471-482. https://doi.org/10.1037/pasoooo209

Espinoza-García, A. (2020). Estilos de socialización parental en una muestra de adolescentes chilenos. Revista de Psicología Clínica con Niños y Adolescentes, 7(1), 46-51. https:// doi.org/10.21134/rpcna.2020.07.1.6

Feixa, C. (2020). Identidad, juventud y crisis: el concepto de crisis en las teorías sobre la juventud. RES, Revista española de sociología, 29(3), 11-26. https://doi.org/g8hr

Fernández, O., Irarrázaval, M., Ortega, B., Carrasco, P., Martínez, P., Rojas, G., \& Mundt, A. (2021). Salud mental primaria para niños, niñas y adolescentes institucionalizados: perspectiva de equipos de salud. Revista Latinoamericana de Ciencias Sociales, Niñez y Juventud, 19(2), 1-19. https://doi.org/10.1160o/rlcsnj.19.2.4175

Gagné, M. (2003). The role of autonomy support and autonomy orientation in prosocial behavior engagement. Motivation and emotion, 27(3), 199-223. https://doi.org/bw4dnj

García, J. M., Lacalle, M., Valbuena, M. C., \& Polaino-Lorente, A. (2019). Relaciones familiares y tiempo compartido y su efecto en el bienestar de los adolescentes. Revista Complutense de Educación, 30(3), 895-915. https://doi.org/10.5209/rced.59562

Gnambs, T., \& Hanfstingl, B. (2016). The decline of academic motivation during adolescence: An accelerated longitudinal cohort analysis on the effect of psychological need satisfaction. Educational Psychology, 36(9), https://doi.org/g8hs

González-Cutre, D., Sierra, A. C., Montero-Carretero, C., Cervelló, E., Esteve-Salar, J., \& Alonso-Álvarez, J. (2015). Evaluación de las propiedades psicométricas de la Escala de Satisfacción de las Necesidades Psicológicas Básicas en General con adultos españoles. Terapia Psicológica, 33(2), 81-92. https://doi.org/g8hv

González-Fuentes, M. B., \& Andrade, P. (2016). Escala de bienestar psicológico para adolescentes. Revista Iberoamericana de Diagnóstico y Evaluación-e Avaliação Psicológica, 2(42), 69-83. https://doi.org/10.21865/RIDEP42_69

Gutiérrez-Carmona, A., Mondaca, C. A., Urzúa, A., \& Wlodarczyk, A. (2020). Can optimism mediate the negative effect of trait anxiety on psychological well-being? 
Revista Interamericana de Psicología/Interamerican Journal of Psychology, 54(2), eg16e916. https://doi.org/10.30849/ripijp.v54i2.916

Hayes, A. F. (2018). Partial, conditional, and moderated moderated mediation: Quantification, inference, and interpretation. Communication Monographs, 85(1), 4-40. https:// doi.org/10.1080/03637751.2017.1352100

Higuita-Gutiérrez, L. F., \& Cardona-Arias, J. A. (2015). Calidad de vida relacionada con la salud en adolescentes: revisión sistemática de las investigaciones publicadas en el periodo 1970-2013. Médicas UIS, 28(1), 23-30.

Huver, R., Otten, R., de Vries, H., \& Engels, R. (2010). Personality and parenting style in parents of adolescents. Journal of Adolescence, 33(3), 395-402. https://doi.org/cghkr5

Keyes, C. L., Shmotkin, D., \& Ryff, C. D. (2002). Optimizing well-being: The empirical encounter of two traditions. Journal of personality and social psychology, 82(6), 1007-1022. https://doi.org/10.1037/0022-3514.82.6.1007

Khaleque, A., \& Rohner, R. P. (2002). Perceived parental acceptance-rejection and psychological adjustment: A meta-analysis of cross-cultural and intracultural studies. Journal of Marriage and Family, 64(1), 54-64. https://doi.org/10.1111/j.1741-3737.2002.00054.x

Krabbenborg, M. A., Boersma, S. N., van der Veld, W. M., Vollebergh, W. A., \& Wolf, J. (2017). Self-determination in relation to quality of life in homeless young adults: Direct and indirect effects through psychological distress and social support. The Journal of Positive Psychology, 12(2), 130-140. https://doi.org/g8h5

Lansford, J. E. (2018). A lifespan perspective on subjective well-being. En E. Diener, S. Oishi, \& L. Tay (Eds.), Handbook of well-being (pp. 1-15). DEF Publishers.

Luna, A. C., Laca, F. A., \& Mejía, J. C. (2011). Bienestar subjetivo y satisfacción con la vida de familia en adolescentes mexicanos de bachillerato. Psicología Iberoamericana, 19(2), 17-26. https://doi.org/10.48102/pi.v19i2.225

Maganto, C., Peris, M., \& Sánchez, R. (2019). El bienestar psicológico en la adolescencia: variables psicológicas asociadas y predictoras. European Journal of Education and Psychology, 12(2), 139-151. https://doi.org/10.30552/ejep.v12i2.279

Mastrotheodoros, S., van der Graaff, J., Deković, M., Meeus, W. H., \& Branje, S. (2020). Parent-adolescent conflict across adolescence: Trajectories of informant discrepancies and associations with personality types. Journal of youth and Adolescence, 49(1), 119-135. https://doi.org/10.1007/s10964-019-01054-7

Meier, L. K., \& Oros, L. B. (2019). Percepción de las prácticas parentales y experiencia de emociones positivas en adolescentes. Revista de Psicología, 8(16), 73-84. 
Minchon, Y. E. (2019). Reflexiona el proceso de socialización e individualización para la construcción de su identidad [Tesis de pregrado, Universidad Nacional de Trujillo]. Repositorio Universidad Nacional de Trujillo. https://bit.ly/3EyZjpE

Molina, M. C., Mateos, A., \& Violant, V. (2015). Estilos de vida saludables de adolescentes acogidos en familia extensa. Revista de Ciencias Sociales, 21(4), 479-493.

Montes, A. J., Cevallos, D. P., \& Alcívar, G.A. (2019). Estilos parentales y calidad de vida familiar en adolescentes con dificultades conductuales: una revisión bibliográfica para el contexto educativo ecuatoriano. Revista Cognosis, 4(3), 169-180.

Morales-Castillo, M., Aguirre-Dávila, E., \& Durán-Urrea, L. (2019). Los contenidos de la formación parental y sus implicaciones en el comportamiento de los adolescentes: elementos desde una revisión. Saúde e Sociedade, 28(3), 224-238. https://doi.org/g8jk

Moreno, J. H., Espada, J. P., \& Gómez, M. I. (2020). Papel de los estilos parentales sobre los problemas internalizantes, externalizantes y de adaptación en niños. Salud mental, 43(2), 73-84. https://doi.org/10.17711/SM.0185-3325.2020.011

Moyano, E., \& Ramos, N. (2007). Bienestar subjetivo: midiendo satisfacción vital, felicidad y salud en población chilena de la región del Maule. Universum, 2(22), 184-200. https://doi.org/10.4067/So718-23762007000200012

Nurius, P. S., Green, S., Logan-Greene, P., \& Borja, S. (2015). Life course pathways of adverse childhood experiences toward adult psychological well-being: A stress process analysis. Child Abuse \& Neglect, 45, 143-153. https://doi.org/f7g84m

Oishi, S., Diener, E., Lucas, R. E., \& Suh, E. M. (2009). Cross-cultural variations in predictors of life satisfaction: Perspectives from needs and values. En E. Diener (Ed.) Culture and well-being (pp. 109-127). Springer. https://doi.org/bn5zt6

Oishi, S., Wyer, R. S., Jr., \& Colcombe, S. J. (2000). Cultural variation in the use of current life satisfaction to predict the future. Journal of Personality and Social Psychology, $78(3)$, 434-445. https://doi.org/10.1037/oo22-3514.78.3.434

Orcasita, L. T., Sevilla, T. M., Acevedo-Velasco, V. E., Montenegro, J. L., Tamayo, M. C., \& Rueda-Toro, J. S. (2020). Apoyo social familiar para el bienestar de hijos gays e hijas lesbianas. Revista Latinoamericana de Ciencias Sociales, Niñez y Juventud, 18(2), 73-95. https://doi.org/10.11600/1692715x.18205

Oyanedel, J. C., Alfaro, J., \& Mella, C. (2015). Bienestar subjetivo y calidad de vida en la infancia en Chile. Revista Latinoamericana de Ciencias Sociales, Niñez y Juventud, 13(1), $313-327$. 
Páez, D., Fernández, I., Campos, M., Zubieta, E., \& Casullo, M. (2006). Apego seguro, vínculos parentales, clima familiar e inteligencia emocional: socialización, regulación y bienestar. Ansiedad y Estrés, 12(2-3), 329-341.

Pons, S. V., García, S., \& Verdecia, A. A. (2020). La identidad personal en los adolescentes: un desafío en el proceso formativo [Ponencia]. III Congreso de Medicina Familiar.

Quintal, G. A., \& Flores, M. M. (2020). Prácticas parentales de crianza y bienestar psicológico en adolescentes. Revista de Psicología de la Salud, 8(1).

Quiroga, F., Capella, C., Sepúlveda, G., Conca, B., \& Miranda, J. (2021). Identidad personal en niños y adolescentes: estudio cualitativo. Revista Latinoamericana de Ciencias Sociales, Niñez y Juventud, 19(2), 1-26. https://doi.org/10.1160o/rlcsnj.19.2.4448

Rodríguez-Menéndez, M. del C., Viñuela-Hernández, M. P., \& Rodríguez-Pérez, S. (2018). Hacia una nueva conceptualización del control parental desde la teoría de la autodeterminación. Teoría de la Educación, Revista Interuniversitaria, 30(1), 179-199. https://doi.org/10.14201/teoreduzo1179199

Ryan, R., \& Deci, E. L. (200o). Self-determination theory and the facilitation of intrinsic motivation, social development, and well-being. American Psychologist, 55(1), 68-78. https://doi.org/10.1037/0003-066X.55.1.68

Ryan, R., Huta, V., \& Deci, E. (2008). Living well: A self-determination theory perspective on eudaimonia. Journal of Happiness Studies, 9(1), 139-170. https://doi.org/cf6rbt

Ryan, R. M., \& Martela, F. (2016). Eudaimonia as a way of living: Connecting Aristotle with self-determination theory. En J. Vittersø (Ed.), Handbook of eudaimonic wellbeing (pp. 109-122). Springer. https://doi.org/10.1007/978-3-319-42445-3-7

Ryff, C. D. (1989). Happiness is everything, or is it? Explorations on the meaning of psychological well-being. Journal of Personality and Social Psychology, 57 (6), 1069-1081. https://doi.org/10.1037/0022-3514.57.6.1069

Ryff, C. D. (1995). Psychological well-being in adult life. Current Directions in Psychological Science, 4(4), 99-104. https://doi.org/10.1111/1467-8721.ep10772395

Ryff, C. D., \& Keyes, C. L. M. (1995). The structure of psychological well-being revisited. Journal of Personality and Social Psychology, 69(4), 719-727. https://doi.org/bsnq6p

Sánchez, M. de los Á. (2019). Adolescentes y bienestar subjetivo: factores protectores y factores de riesgo. [Tesis de Maestría, Universidad de Córdoba]. Helvia, Repositorio Institucional de la Universidad de Córdoba. http://hdl.handle.net/10396/18475

Schoenmaker, C., Juffer, F., van IJzendoorn, M. H., Linting, M., van der Voort, A., \& Bakermans-Kranenburg, M. J. (2015). From maternal sensitivity in infancy to adult 
attachment representations: A longitudinal adoption study with secure base scripts. Attachment \& Human Development, 17(3), 241-256. https://doi.org/gckgf5

Steinmayr, R., Wirthwein, L., Modler, L., \& Barry, M. M. (2019). Development of subjective well-being in adolescence. International Journal of Environmental Research and Public Health, 16(19), 3690. https://doi.org/10.339o/ijerph1619369o

Tirado, K. P., \& Quezada, E. E., (2019). Estilos parentales y percepción de autoeficacia académica en estudiantes de 10 a 13 años del sur del DMQ. Analysis. Claves de Pensamiento Contemporáneo, 24, 45-56.

Torres, A. (2019). Los estilos de crianza y su papel e impacto en el desarrollo infantojuvenil [Tesis de pregrado, Universitat Abat Oliba CUE]. CUE Repositorio Institucional. http://hdl.handle.net/10637/11049

van Dierendonck, D. (2004). The construct validity of Ryff's Scales of Psychological Wellbeing and its extension with spiritual well-being. Personality and Individual Differences, 36(3), 629-643. https://doi.org/10.1016/So191-8869(03)oo122-3

Vergaray, F., \& Benavides, G. A. (2020). Relación entre funcionamiento familiar y bienestar psicológico en estudiantes adolescentes de instituciones educativas de Lima [Tesis de pregrado, Universidad Peruana Unión]. Repositorio de Tesis Universidad Peruana Unión. http://hdl.handle.net/20.500.12840/3108

Viejo, C., \& Ortega-Ruiz, R. (2015). Cambios y riesgos asociados a la adolescencia. Psychology, Society \& Education, 7(2), 109-118. https://doi.org/10.25115/psye.v7i2.527

Villarejo, S., Martínez-Escudero, J. A., \& Garcia, O. F. (2020). Estilos parentales y su contribución al ajuste personal y social de los hijos. Ansiedad y Estrés, 26(1), 1-8. https://doi.org/10.1016/j.anyes.2019.12.001

Wehmeyer, M. L. (2014). Self-determination: A family affair. Family Relations, 63(1), 178184. https://doi.org/10.1111/fare.12052

Wood, D., Crapnell, T., Lau, L., Bennett, A., Lotstein, D., Ferris, M., \& Kuo, A. (2018). Emerging adulthood as a critical stage in the life course. En N. Halfon, C. Forrest, Lerner R., \& E. Faustman (Eds.), Handbook of life course health development (pp. 123-143). Springer. https://doi.org/10.1007/978-3-319-47143-3-7

Yu, S., Levesque-Bristol, C., \& Maeda, Y. (2018). General need for autonomy and subjective well-being: A meta-analysis of studies in the US and East Asia. Journal of Happiness Studies, 19(6), 1863-1882. https://doi.org/10.1007/s10902-017-9898-2 